\title{
Consciousness, Reductionism and the Explanatory Gap: Investigations in Honor of Rudolf Carnap
}

\author{
Leon de Bruin • Albert Newen
}

Received: 10 August 2010 /Accepted: 15 August 2010 /

Published online: 17 September 2010

(C) Springer Science+Business Media B.V. 2010

The 'Carnap Lectures' feature a series of lectures by an internationally renowned scholar in philosophy in combination with an international graduate workshop. The lectures are dedicated to Rudolf Carnap, who was born in the Ruhr-Area not far from Bochum (Wuppertal) and began his philosophical career in Germany before emigrating to the United States. The Carnap Lectures were initiated and organized by Albert Newen in 2008, and have become an annual event at the Institute of Philosophy of the Ruhr-University of Bochum. In 2008 the Carnap lectures were presented by Alva Noë (Berkeley/New York), who talked about 'Perception, Knowledge and Performance', and in 2009 John Perry (Stanford) discussed various topics related to 'Meaning and the Self'.

The Carnap Lectures 2010 focused on the work of David Papineau, who presented his views on several important topics in the philosophy of mind: (i) the 'explanatory gap', (ii) the dualist intuition, (iii) the phenomenal concept strategy, (iv) reductionism and (v) mental causation. His keynote article in this special issue deals in particular with the first two topics: the explanatory gap and the dualist intuition. Many contemporary philosophers of mind believe that science leaves us with an explanatory gap - that even after we know everything that science can tell us about the conscious mind and the brain, their relationship still remains mysterious. In 'What exactly is the explanatory gap?', Papineau argues that this view is mistaken. Science does tell us everything there is to know about mental phenomena, and the impression of a gap is due to nothing but an intuitive conviction that dualism is true and materialism false. The question is how we should explain this dualist intuition, and how we can learn to live with it. Papineau's first aim is to show that a posteriori explanations have no disadvantages vis-à-vis a priori explanations when it comes to explanatory power. He proposes that "given the identity of pain with C-fibre firings, we can use the facts that $\mathrm{C}$-fibre firings are themselves caused by bodily damage and gives rise to avoidance behaviour to explain why pain has those characteristics. [...] a

L. de Bruin $(\triangle) \cdot$ A. Newen

Institut für Philosophie II, Ruhr-Universität Bochum, Universitätsstr. 150, 44801 Bochum, Germany e-mail: leon.debruin@rub.de 
posteriori mental-material identities seem no less explanatory than a priori derivable identities." (Papineau, this volume, p.***). Papineau concedes that even if we know mind-brain identity claims to be true, we are nevertheless inclined to think that there must be some kind of explanatory gap. For if we really would accept the truth of these identity claims, we would reject Zombie thought-experiments-just as we would reject someone's claim that Cicero is a famous orator and Tully is not, if we know that Cicero and Tully are identical. Why is the dualist intuition so persistent, despite the continuous discovery of new mind-brain identities? Papineau discusses several possibilities that might explain this (for example, the fact that most human beings are raised as dualists since dualism is deeply anchored in many cultures) and concludes that the dualist intuition is probably the 'product of multiple factors pushing in the same direction' (ibid., $\mathrm{p}^{* * *}$ ). In the end, however, the question what causes the dualist intuition is an empirical question that can only be answered by empirical means. The philosophical message is that this is all there is to explain, since the explanatory gap itself does not exist.

The Carnap Lectures 2010 also featured an international graduate workshop with the title 'Consciousness, Reductionism and the Explanatory Gap'. A select number of graduate students were invited to present a paper during this workshop, on the basis of a peer-review process by an international board of distinguished philosophers. Six of these papers are included in this volume.

In 'Two levels of metacognition', Arango argues that we must distinguish between high-level and low-level metacognition in order to do justice to this complex phenomenon. Both levels have a different structure, a different content and a different function within the overall cognitive architecture. Arango reviews two rivaling theories of metacognition and shows that they are seriously limited, in the sense that they focus on only one of these levels. He then presents his own account, according to which high-level metacognition should be characterized in terms of rationality (the agent uses concepts and theories to interpret his/her own behavior), and low-level metacognition in terms of control (the agent exploits epistemic feelings to adjust his/her cognitive activities). Finally, Arango explores three types of interaction between high-level and low-level metacognition.

Fazekas' 'Cognitive Architecture and the Epistemic Gap' puts forward an explanation for the occurrence of the epistemic gap, and defends physicalism against anti-physicalist arguments without relying on phenomenal concepts. Instead of concentrating on conceptual features, Fazekas shifts his focus to the special characteristics of experiences themselves. He argues that certain sensory representations lack constituent structure, and are therefore unanalyzable from a functional perspective. Together with the encapsulated nature of the low-level complex processes that give rise to unstructured sensory representations, these features readily explain those peculiarities of phenomenal consciousness which are usually taken to pose a serious problem for contemporary physicalism. Fazekas concludes that if those concepts which are related to the phenomenal character of conscious experience are special in any way, their speciality is derivative and we can account for them in terms of the cognitive and representational features he proposes.

Fisher, in 'Quine's Behaviorism and Linguistic Meaning', argues against the idea that Quine is committed to an 'illicit' behaviorist account of linguistic meaning. Fisher claims that if one sets out to dismiss Quine's arguments for internal-meaning 
skepticism, this dismissal cannot be based on a denial of the three following claims often associated with meaning behaviorism: (i) language-users associate mental ideas with their linguistic expressions, (ii) a language-user can have a private theory of linguistic meaning which guides his or her use of language, and (iii) language learning relies on innate mechanisms. Fisher argues that Quine could grant all three of these claims, and he shows that Quine's behaviorism should be viewed as a largely uncontroversial aspect of his evidential empiricism.

In 'Is Imagination Introspective', Reuter discusses the question whether we should think of imagination as a species of introspection. Consensus has it that in sensory imagination the focus is on the imagined objects, not on the imaginative states themselves. Therefore, imagination is not a species of introspection, and the introspection of imaginative states is thought to be an additional cognitive ability. Reuter, however, argues that we should classify at least some forms of imagination as introspective. For example, in many cases in which we sensorily imagine a certain object in front of us, we are aware that this object is not really where we imagine it to be. So it looks as if in these cases of imagination, we are aware of the mere appearance of the imagined object, and hence introspection is a constitutive part of imagination. Reuter defends this thesis of introspective imagination against the objection that young children imagine without yet understanding the concept of experience.

Bernal Velasquez argues in 'Materialism and the Subjectivity of Experience' against the idea that for something to be real it must (at least in principle) be accessible from an intersubjective perspective. He claims that the exclusively subjective access to phenomenal contents can be explained by the very particular nature of the epistemological relation holding between a subject and his own mental states. According to Bernal Velasquez' account, this kind of subjectivity does not compel us to deny the possibility that phenomenal contents are ontologically objective properties.

In 'Restrictive Materialism and the Propositional Attitudes', Holman presents a version of materialism position according to which there is a restricted domain in which the application of the folk ontology remains secure. Based on evidence of developmental uniformity and cross-cultural ubiquity of beliefs and desires, Holman takes issue with Churchland's elimative materialism and argues that the ontology but not the principles of folk psychology may be incorrigible. Given that the domain of psychology is how humans think, Holman claims that beliefs and desires will continue to earn their rightful place in the ontology of any future psychology.

We close with a word of thanks to the philosophers who participated in the intensive peer-review process that preceded this special issue of the Carnap Lectures 2010. Their assistance in selecting the six best papers out of twenty-five high-quality submissions was invaluable, as was their help in the second round of review and their comments on the revised papers. All papers in this volume have greatly benefited from their constructive criticism. We also wish to express our gratitude to Michael Esfeld and Stephan Hartmann and their research groups. The Carnap Lectures really profit from the close cooperation between the departments of philosophy in Bochum, Lausanne and Tilburg.

Bochum, the 7th of August 2010

Leon de Bruin and Albert Newen 